\title{
Direct Imaging of the ac Component of Pumped Spin Polarization with Element Specificity
}

\author{
S. Pile®, ${ }_{1}^{1}$ M. Buchner, ${ }^{1}$ V. Ney®, ${ }^{1}$ T. Schaffers $\odot,{ }^{1, \dagger}$ J. Lumetzberger, ${ }^{1}$ K. Lenz $\odot,{ }^{2}$ R. Narkowicz, ${ }^{2}$ \\ J. Lindner, ${ }^{2} \mathrm{H}$. Ohldag $\odot,{ }^{3,4, \ddagger}$ and A. Ney®${ }^{1, *}$ \\ ${ }^{1}$ Institute of Semiconductor and Solid State Physics, Johannes Kepler University, Altenberger Strasse 69, \\ Linz 4040, Austria \\ ${ }^{2}$ Helmholtz-Zentrum Dresden-Rossendorf, Institute of Ion Beam Physics and Materials Research, Bautzner \\ Landstrasse 400, Dresden 01328, Germany \\ ${ }^{3}$ Stanford Synchrotron Radiation Laboratory, SLAC National Accelerator Laboratory, Menlo Park, \\ California 94025, USA \\ ${ }^{4}$ Department of Physics, University of California Santa Cruz, Santa Cruz, California 95064, USA
}

(Received 11 May 2020; revised 29 June 2020; accepted 13 July 2020; published 1 September 2020)

\begin{abstract}
Spin pumping in a ferromagnet-nonferromagnet heterostructure is directly imaged with spatial resolution as well as element selectivity. The time-resolved detection in scanning transmission x-ray microscopy allows us to directly probe the spatial extent of the ac spin polarization in Co-doped $\mathrm{ZnO}$, which is generated by spin pumping from an adjacent permalloy microstrip. Comparing the relative phases of the dynamic magnetization component of the two constituents is possible and found to be close to antiphase. The correlation between the distribution of the magnetic excitation in the permalloy and the Co-doped $\mathrm{ZnO}$ reveals that laterally there is no one-to-one correlation. The observed distribution is rather complex, but integrating over larger areas demonstrates that the spin polarization in the nonferromagnet extends laterally beyond the region of the ferromagnetic microstrip. Therefore, the observations are better explained by a local spin pumping efficiency and a lateral propagation of the ac spin polarization in the nonferromagnet over the range of a few micrometers.
\end{abstract}

DOI: 10.1103/PhysRevApplied.14.034005

\section{INTRODUCTION}

In spintronics the generation and manipulation of pure spin currents is in the focus of research activities. Amongst the utilized fundamental effects is spin pumping where a precessing magnetization of a ferromagnet being at ferromagnetic resonance (FMR) transfers angular momentum to an adjacent nonferromagnetic layer [1]. The transfer of angular momentum into the nonferromagnetic layer can be described as a spin current. The pumped spin current has

\footnotetext{
*andreas.ney@jku.at

$\dagger$ Present address: NanoSpin, Department of Applied Physics, Aalto University School of Science, P.O. Box 15100, FI-00076 Aalto, Finland.

$\$$ Present addresses: Advanced Light Source, Lawrence Berkeley National Laboratory, Berkeley, CA 94720, USA and Department of Material Sciences and Engineering, Stanford University, Stanford, CA 94305, USA.

Published by the American Physical Society under the terms of the Creative Commons Attribution 4.0 International license. Further distribution of this work must maintain attribution to the author(s) and the published article's title, journal citation, and DOI.
}

a dc and an ac component corresponding to the reduction in the projection of the magnetization at FMR and the dynamic high-frequency magnetization, respectively [2-4]. Usually, spin pumping is electrically detected via the inverse spin Hall effect (ISHE) inside the nonferromagnetic layer that makes conducting high- $Z$ materials such as Pt advisable for easy detection $[3,5,6]$; however, for few other materials like semiconducting Ge [7], conducting $\mathrm{SrRuO}_{3}$ [8] and $\mathrm{ZnO}$ [9] spin pumping could be detected via the ISHE as well. From the perspective of suitable materials, a more versatile approach is to detect the presence of spin pumping via the increased FMR linewidth $[4,10,11]$. It is nowadays consensus between experiment and theory that the flow of angular momentum from the ferromagnet into the nonferromagnet represents another Gilbert-like damping mechanism. Such spin pumping heterostructures allow different nonferromagnetic materials to be used without the restriction of the ISHE as the detection channel. However, the advantages of using nonconducting materials have been pointed out for the ferromagnet [11], as well as the nonferromagnet [4]. Insulating nonferromagnets have the advantage that the increased magnetic damping is less influenced by other mechanisms like eddy-current damping; see Ref. [4] for a recent overview. 
A major drawback in general is that most of the experimental methods used so far detect spin pumping only indirectly, no matter if they rely on the ISHE or the increased FMR linewidth. In addition, in most approaches only the dc component of the pumped spin polarization inside the nonferromagnet is measured; however, the ac component, which was theoretically predicted to be rather large compared to the dc component [2], was also observed experimentally in an Yttrium-iron-garnet/Pt heterostructure [12] as well as in permalloy (Py)/Pt heterostructures via the ISHE $[3,13]$.

It is the aim of the present work to investigate the ac component of the pumped spin polarization directly inside the nonferromagnet with ultimate spatio-temporal resolution and elemental selectivity. This is achieved by using a time-resolved detection scheme in combination with a scanning transmission x-ray microscope (STXM) [14]. In combination with a microwave excitation this STXM-FMR setup allows us to excite the FMR of a ferromagnetic microstructure in contact with an adjacent nonferromagnetic material, which was chosen to be insulating, i.e., inaccessible by the ISHE. This combination allows us to utilize the known lateral resolution of a few tens of nanometers of the STXM to investigate the lateral extent of the generated spin polarization. This basic approach has already been shown to be feasible for electrical spin injection into a nonferromagnetic metal [15] and very recently to also probe the ac component in $\mathrm{Py} / \mathrm{Cu}$ heterostructures [16], which was evidenced before using time-resolved measurements without spatial resolution [17]. The time-resolved detection scheme based on the internal picosecond time structure of the synchrotron allows us to sample dynamics up to the gigahertz regime, i.e., FMR frequencies [14]. Finally, STXM FMR also allows for probing the magnetic properties with element selectivity, since the contrast mechanism is based on the $\mathrm{x}$ ray magnetic circular dichroism (XMCD) [18]. In view of this, the dynamic out-of-plane component of the precessing magnetization during FMR can be measured. Therefore, the magnetic response of the driving ferromagnet and the pumped spin polarization inside the nonferromagnetic material can be probed directly and independently. We find a pumped ac spin polarization that is close to antiphase to the ferromagnet. Its spatial extent is not homogeneous and it extends laterally beyond the region of the ferromagnet.

The system of choice for the present study is a heterostructure consisting of Py in contact with $\mathrm{Zn}_{0.5} \mathrm{Co}_{0.5} \mathrm{O}$ ( $50 \% \mathrm{Co}: \mathrm{ZnO})$, a system where the presence of spin pumping has been indirectly evidenced before via increased Gilbert damping in FMR [19]. Under the experimental conditions of the STXM FMR, i.e., at ambient temperature, $50 \% \mathrm{Co}: \mathrm{ZnO}$ is known to be weakly paramagnetic [19] and highly insulating. In addition the $L_{3}$ edge of Co is in close vicinity in terms of necessary photon energy of the
Fe and Ni $L_{3}$ edges so that both Py and the Co:ZnO can be conveniently studied with the identical STXM setup.

Single films of $\mathrm{Py}, 50 \% \mathrm{Co}: \mathrm{ZnO}$, and heterostructures thereof are fabricated on $c$-plane sapphire substrates as well as SiN membranes on highly insulating Si substrates by using reactive magnetron sputtering at a process pressure of $4 \times 10^{-3}$ mbar in the same ultrahigh vacuum chamber with a base pressure of $2 \times 10^{-9}$ mbar. For the $50 \% \mathrm{Co}: \mathrm{ZnO}$ layer, a metallic composite target is used, an $\mathrm{Ar}: \mathrm{O}_{2}$ ratio of 10:1 standard cubic centimeters per minute $(\mathrm{sccm})$ is used as a process gas, and the substrate temperature is kept at $294{ }^{\circ} \mathrm{C}$, which are optimized growth conditions for $50 \% \mathrm{Co}: \mathrm{ZnO}[19,20]$. Py with a typical thickness of $20 \mathrm{~nm}$ is fabricated at room temperature using $10 \mathrm{sccm}$ of only Ar as a process gas. To prevent oxidation, all Py films are subsequently covered with a $6 \mathrm{~nm}$ thick Al capping layer grown under identical conditions using pulsed laser deposition. The feasibility of FMR excitation of a Py microstrip using a micro resonator atop of a $\mathrm{Co}: \mathrm{ZnO}$ film is tested using a $50 \% \mathrm{Co}: \mathrm{ZnO}$ film grown on highly insulating Si substrate under identical conditions as above. In a second step a $1 \times 5 \mu \mathrm{m}^{2}$ Py microstrip with a thickness of $20 \mathrm{~nm}$ and an Al cap is fabricated using standard $e$-beam lithography (EBL). In a final step a planar micro resonator (see Ref. [21,22]) with a total Au-plating thickness of $600 \mathrm{~nm}$ is fabricated by optical lithography. For the STXM-FMR measurements, a $4 \times 4 \mathrm{~mm}^{2}$ and 100 $\mathrm{nm}$ thick $50 \%$ Co: $\mathrm{ZnO}$ film is grown on a commercial $200 \mathrm{~nm}$ thick $0.25 \times 0.25 \mathrm{~mm}^{2}$ SiN membrane on a highly insulating $5 \times 10 \mathrm{~mm}^{2}$ Si substrate using shadow masking. Again, an identical $1 \times 5 \mu \mathrm{m}^{2}$ Py microstrip is fabricated using EBL. In a final step a strip-type resonant structure with a total Au-plating thickness of $600 \mathrm{~nm}$ is fabricated by optical lithography; see Ref. [23] for details.

FMR precharacterization of the chosen materials for the spin pumping heterostructure is done in a home-built broadband FMR setup, as reported before [19]. For that, an unstructured $50 \% \mathrm{Co}: \mathrm{ZnO} / \mathrm{Py}$ heterostructure and a single Py film grown on $c$-sapphire substrates are measured using a microwave short adapted after Ref. [24] in a frequency range from 3-10 GHz, as shown in Fig. 1(a). While the frequency dependence of the resonance field $\left(\mu_{0} H_{\text {res }}\right)$ is identical for both samples, the frequency-dependent peak-to-peak linewidth $\left(\mu_{0} H_{\mathrm{pp}}\right)$ exhibits a linear behavior with an increased slope for the heterostructure compared to the Py film. This leads to a Gilbert damping parameter of $\alpha_{\text {Py }}=(5.5 \pm 0.3) \times 10^{-3}$ for Py and an increased Gilbert damping parameter of $(8.0 \pm 0.3) \times 10^{-3}$ for the heterostructure. This has already been discussed before as experimental evidence that angular momentum transfer via spin pumping is possible in $\mathrm{Co}: \mathrm{ZnO} / \mathrm{Py}$ heterostructures [19]. The direct experimental evidence of the presence of a pumped spin polarization in the nonferromagnet is however lacking based on Fig. 1(a) only and is inaccessible for the detection via the ISHE. 


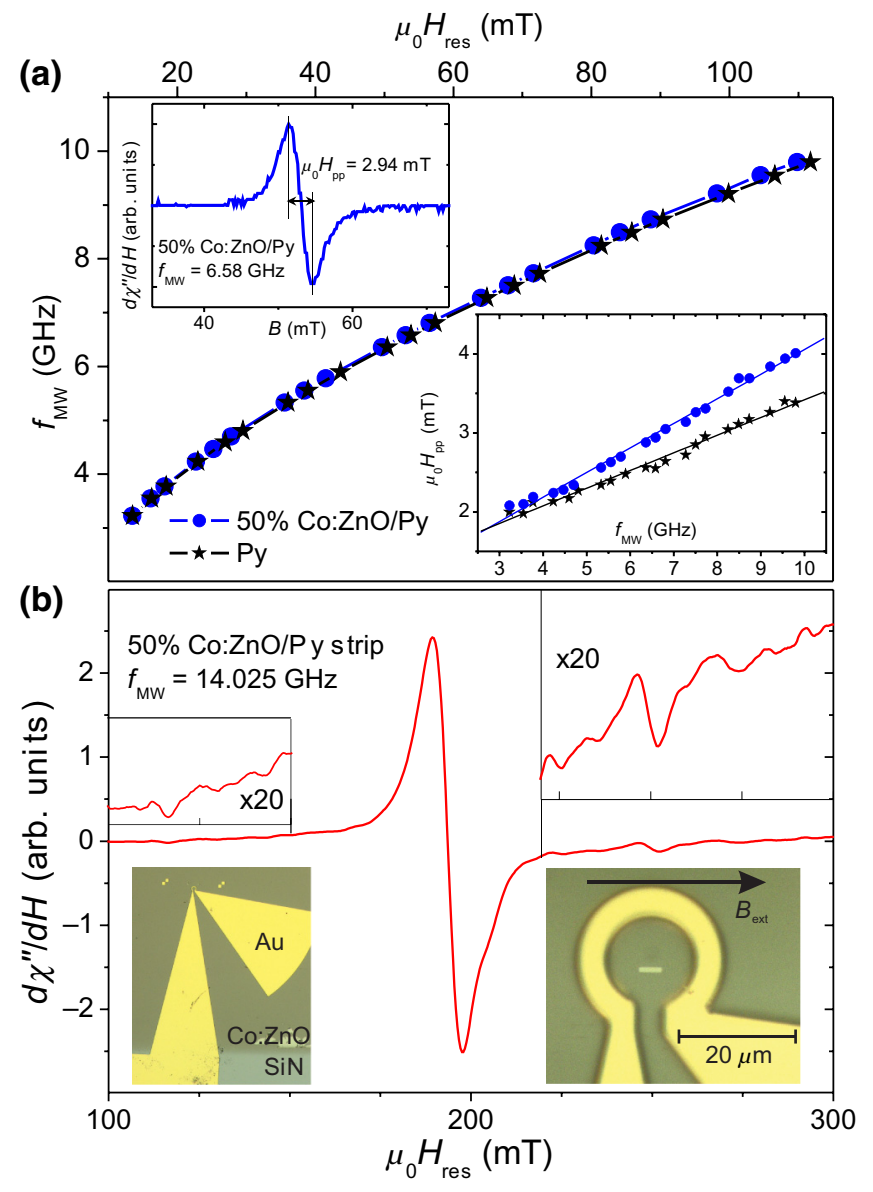

FIG. 1. (a) Broadband FMR measurements of the resonance field $\mu_{0} H_{\text {res }}$ versus microwave frequency $f_{\mathrm{MW}}$ for a bare Permalloy (Py) film (stars) and a 50\% Co: $\mathrm{ZnO} / \mathrm{Py}$ heterostructure both grown on sapphire. The upper inset shows the FMR line and corresponding linewidth $\mu_{0} H_{\mathrm{pp}}$ at $f_{\mathrm{MW}}=6.58 \mathrm{GHz}$. The lower inset summarizes the frequency-dependent linewidth. (b) FMR spectrum of the $50 \% \mathrm{Co}: \mathrm{ZnO} / \mathrm{Py}$ strip sample measured at 14.025 $\mathrm{GHz}$; the regions of the spin-wave excitations are enlarged. The insets show optical images (overview and closeup) of the planar micro resonator with the strip sample inside.

To fabricate a spin pumping heterostructure suitable for the STXM-FMR detection scheme, we have to prove in a first step that the micro resonator scheme can operate on top of a contingent $50 \% \mathrm{Co}: \mathrm{ZnO}$ film. For this, we study a $\mathrm{Co}: \mathrm{ZnO}$ film grown on highly insulating $\mathrm{Si}$ substrate with a Py microstrip on top using a planar micro resonator optimized for $14.025 \mathrm{GHz}$, as shown in the images in Fig. 1(b). The conventional FMR spectrum in Fig. 1(b) exhibits a clear FMR main mode close to $200 \mathrm{mT}$ as well as spin-wave excitations above and below the main mode (enlarged by a factor of 20), which have been reported before [25]. The fact that the planar resonator is sensitive enough to detect even these spin-wave modes implies that the $50 \% \mathrm{Co}: \mathrm{ZnO}$ film is sufficiently insulating (i.e., above $3 \mathrm{k} \Omega \mathrm{cm}$ ) so that the micro resonator is not shorted out and maintains a high sensitivity. This is in agreement with the fact that nominally $100 \mathrm{~nm}$ thick $50 \%$ Co: $\mathrm{ZnO}$ films on sapphire typically exhibit a resistance in the $\mathrm{G} \Omega$ range.

In a next step it is verified that the structural and magnetic properties of $50 \% \mathrm{Co}: \mathrm{ZnO}$ do not significantly change when growing on the $\mathrm{SiN}$ membrane compared to $c$-sapphire. In Fig. 2(a) we show a symmetric $\omega-2 \theta$ x-ray diffraction (XRD) scan using $\mathrm{Cu} K_{\alpha}$ radiation. The $\mathrm{Si}(004)$ reflection of the highly insulating Si substrate supporting the SiN membrane is nicely visible as well as the typical wurtzite $\mathrm{ZnO}(002)$ reflection [26]. Its position is comparable to $\mathrm{Co}: \mathrm{ZnO}$ grown on $c$-sapphire; however, the full-width at half maximum (FWHM) $0.39^{\circ}$ is slightly larger than that for the best films grown on sapphire where a FWHM of down to $0.18^{\circ}$ can be achieved [26]. The wideangle XRD scan is devoid of other reflection, ruling out the formation of secondary crystalline phases. Thus, the $50 \% \mathrm{Co}: \mathrm{ZnO}$ film growth is highly $c$-oriented not only on sapphire but on the SiN membranes also. In Fig. 2(b) we show superconducting quantum interference device (SQUID) magnetometry measurements following a standard protocol; see Ref. [26]. Note that all magnetizaiton data have been corrected for the diamagnetic signal of the repective substrate. For better comparability all resulting magnetization values $(M)$ were further divided by the magnetization value recorded at $T=2 \mathrm{~K}$ and $H=5 \mathrm{~T}[\mathrm{M}$ $(2 \mathrm{~K}, 5 \mathrm{~T})]$ for the respective sample. The $M(H)$ curve at $2 \mathrm{~K}$ exhibits for both samples the typical, narrowly opened and vertically shifted hysteresis that is indicative of uncompensated antiferromagnetism [20,26]. More importantly, for STXM-FMR measurements, which are conducted at room temperature only, the $M(T)$ measurements recorded at $10 \mathrm{mT}$ under field heated $(\mathrm{FH})$, field cooled (FC), and zero field cooled (ZFC) conditions reveal that the magnetic order is restricted to below $25 \mathrm{~K}$ and no evidence of the formation of Co metallic clusters can be derived by SQUID magnetometry. It should be noted that, for $60 \% \mathrm{Co}: \mathrm{ZnO}$ grown on $\mathrm{SiN}$ membranes, the situation is different and a clear superparamagnetic blocking behavior typical for the formation of metallic Co precipitates is seen by SQUID magnetometry. This is corroborated by conventional STXM measurements around the $\mathrm{Co}_{3}$ edge, where a heterogeneous appearance of the $\mathrm{Co}: \mathrm{ZnO}$ film is found for $60 \% \mathrm{Co}: \mathrm{ZnO}$, which is absent for the $50 \% \mathrm{Co}: \mathrm{ZnO}$ (not shown).

The STXM-FMR measurements themselves have been described in detail in Refs. [14,23,27,28]. In brief, the microwave frequency is phase locked to a high harmonic of the internal time structure of the synchrotron to record the $\mathrm{x}$-ray transmission at normal incidence using circular polarized light at six different relative phases between the microwave excitation and arrival of the x-ray light pulse. Every other revolution of the electron bunches a fast PIN diode switches the microwave power off, so that the six phases are recorded once with precessing magnetization 


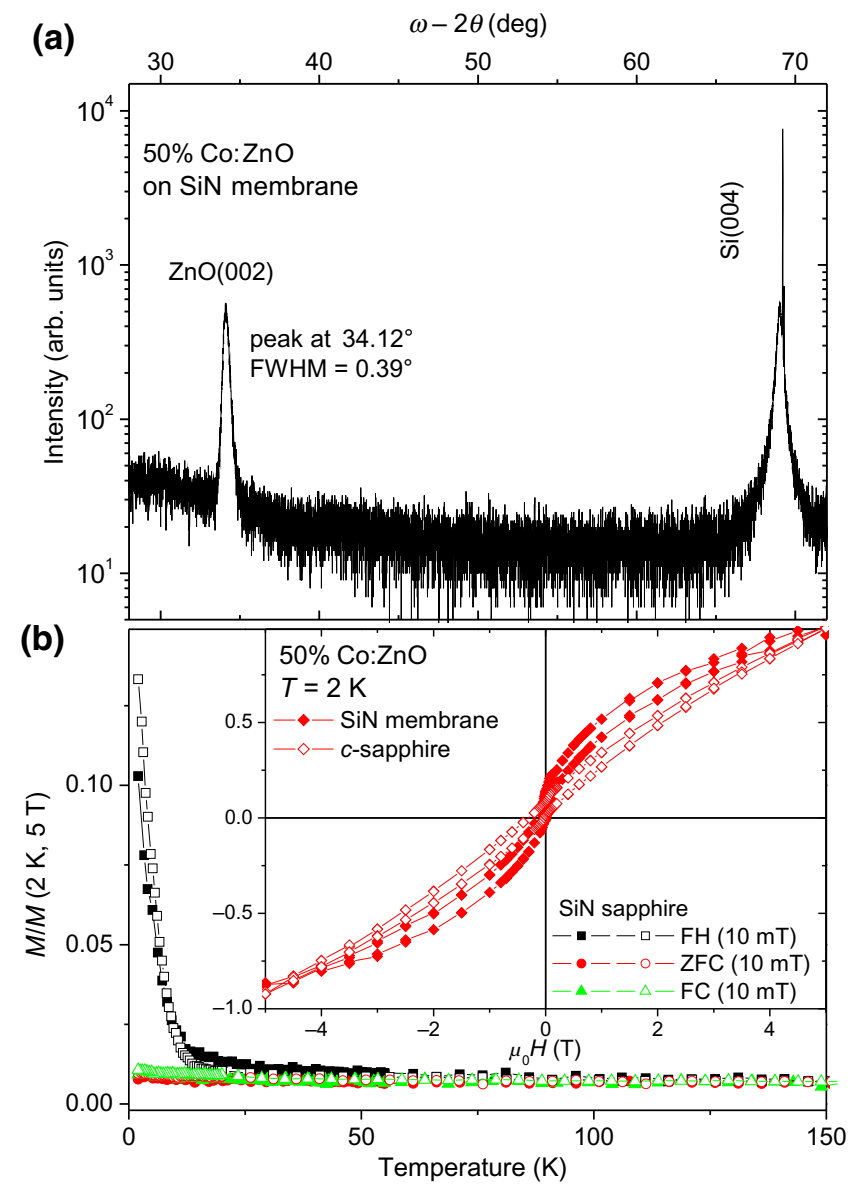

FIG. 2. (a) XRD $\omega-2 \theta$ scan of a $50 \% \mathrm{Co}: \mathrm{ZnO}$ film grown on the $\mathrm{SiN}$ membrane. (b) Integral magnetic characterization of $50 \%$ $\mathrm{Co}: \mathrm{ZnO}$ films grown on sapphire (open symbols) as well as on a SiN membrane (filled symbols). The normalized $M(T)$ curves are shown under different cooling conditions together with the normalized $M(H)$ curves at $2 \mathrm{~K}$ in the inset.

(microwave on) and once with nonprecessing magnetization (microwave off). By means of the XMCD effect, the difference between the images with microwave on and off is proportional to the out-of-plane magnetization component and the six phases thus reflect one full precession cycle of the magnetization in FMR. In Fig. 3 the dynamic magnetic contrast of a Py microstrip on top of a $50 \%$ $\mathrm{Co}: \mathrm{ZnO}$ film is shown for a microwave frequency of 9.447 $\mathrm{GHz}$ and an external in-plane magnetic field $B_{\text {ext }}$ of 96 $\mathrm{mT}$; see the inset of Fig. 1(b). Six consecutive phases are recorded once at the $\mathrm{Ni} L_{3}$ edge (top images) and once at the Co $L_{3}$ edge (bottom images). The region of the Py strip that could be safely derived from the time-integral $Z$-contrast image at the $\mathrm{Ni} L_{3}$ edge (see the white frame in the inset of Fig. 4) is marked by the black frames in Fig. 3. It is obvious that at the $\mathrm{Ni} L_{3}$ edge the quasiuniform FMR excitation of the Py microstrip can be detected; see Ref. $[27,28]$. Outside the region of the strip, little dynamic

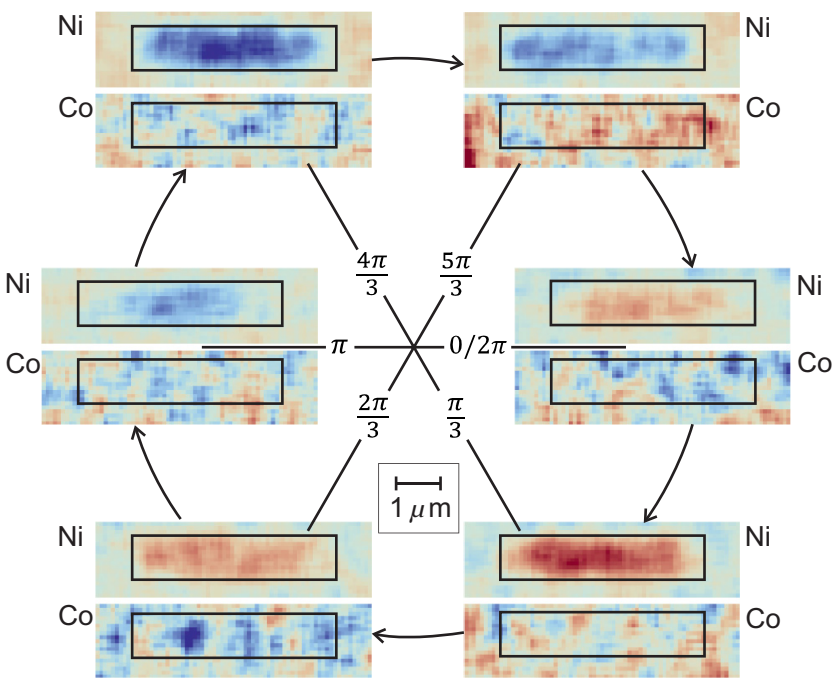

FIG. 3. Dynamic magnetic contrast at six relative phases between the microwave excitation and x-ray pulse for a $50 \%$ $\mathrm{Co}: \mathrm{ZnO} / \mathrm{Py}$ heterostructure at $f_{\mathrm{MW}}=9.447 \mathrm{GHz}$ and $B_{\text {ext }}=96$ $\mathrm{mT}$ recorded at the $\mathrm{Ni}$ (top images) as well as the Co (bottom images) $L_{3}$ edges. The region of the Py strip is marked by the black rectangle.

magnetic contrast is visible. At the $\mathrm{Co}_{3}$ edge the overall magnetic contrast is much weaker and thus appears more noisy. We can see that the Ni signal is strongest at phases $\pi / 3$ (mainly red color) and $4 \pi / 3$ (mainly blue color), while the Co signal tends to be of opposite contrast, which is best seen at phases $2 \pi / 3$ and $5 \pi / 3$, i.e., the dynamic magnetization of $\mathrm{Ni}$ and $\mathrm{Co}$ appear to be roughly at antiphase.

For a more in-depth analysis of the observed dynamic magnetic contrast, the available magnetic information can be visualized differently. As done before [23,27], the magnetic contrast can be averaged over a region of interest and plotted versus (relative) time. For better clarity, one full precession period has been duplicated so that two identical consecutive cycles are shown. The result can be seen in Fig. 4 for the $\mathrm{Ni}$ (a) and the $\mathrm{Co}$ (b) $L_{3}$ edge for two different regions of interest: the area of the Py strip (black squares) and the region outside the strip (red circles). The data can be fitted with a sine function shown by the lines. At the $\mathrm{Ni}$ edge the dynamic magnetic contrast is nicely visible in the region of the strip. If the region surrounding the strip is averaged, only a very small signal is visible. It is important to note that the microwave excitation itself always creates a nonmagnetic effect also in the background [23] that is also seen here. This contrast modulation is directly and nonresonantly created by the microwave and, thus, serves as a reference phase of the driving microwave. Now the phase shift is more clearly visible: the quasiuniform FMR excitation of the Py strip is phase shifted by approximately $75^{\circ}$ with respect to the microwave, which is typical for any driven system close to resonance, e.g., the driven harmonic 


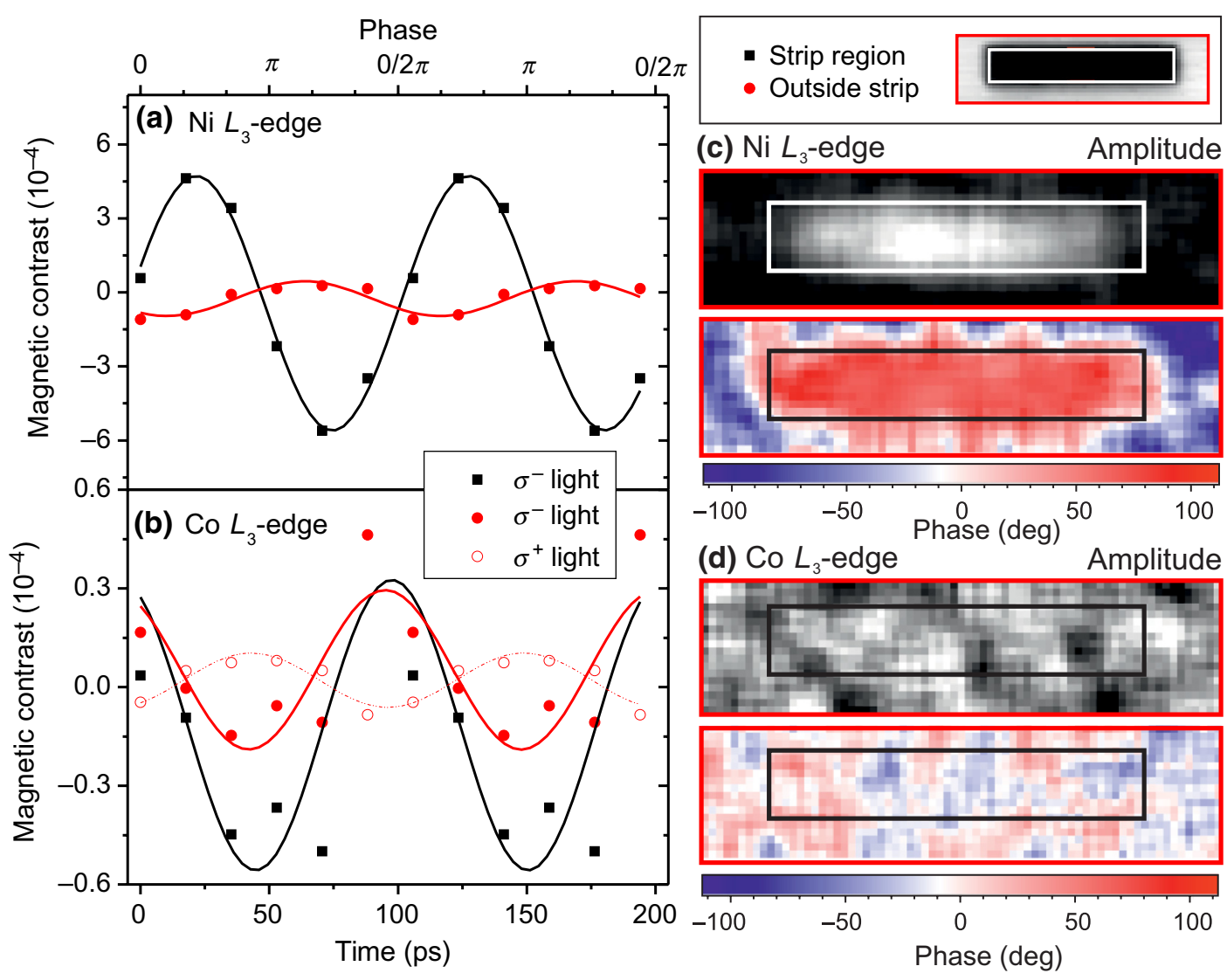

FIG. 4. (a) Integrated dynamic magnetic contrast over two precession cycles recorded for the main FMR mode at $f_{\mathrm{MW}}=9.447 \mathrm{GHz}$ and $B_{\text {ext }}=96 \mathrm{mT}$ recorded at the Ni $L_{3}$ edge for the region of the strip (black) or the region outside the strip (red), as marked in the Z-contrast image of the Py strip in the inset. (b) Corresponding integrated dynamic magnetic contrast at the Co $L_{3}$ edge recorded with $\sigma^{-}$light; the open symbols correspond to $\sigma^{+}$light. The corresponding point-by-point analysis (for details, see the text) of resulting local amplitude and phase derived at the $\mathrm{Ni}$ (c) and $\mathrm{Co}$ (d) $L_{3}$ edges. The positions of the $1 \times 5 \mu \mathrm{m}^{2}$ large Py strip is indicated by the white and black frames.

oscillator near the resonance is phase shifted by $90^{\circ}$ with respect to the periodic external force.

At the Co $L_{3}$ edge shown in Fig. 4(b) the integrated dynamic magnetic contrast shows significant differences compared to the Ni edge: (i) the overall amplitude is about a factor of ten smaller, (ii) the phase at the Co edge is different from both the background and the Ni edge, and (iii) the contrast is also visible outside the strip region. Observation (i) is understandable since in spin pumping transfer of angular momentum across a (nonideal) interface is presumably suppressed by spin scattering. Observation (ii) demonstrates the important fact that Co has a phase of its own. The phase shift of Co with regard to the Ni background, i.e., the driving microwave, is about $45^{\circ}$, which implies that the observed contrast is not a direct effect of the microwave. Ni and Co have a relative phase of about $135^{\circ}$, i.e., close to antiphase, which is also suggested by the almost opposite amplitudes visible in Figs. 4(a) and 4(b), respectively. This observation could by understood by adapting common arguments for the conservation of angular momentum for the static part of the pumped spin polarization as, e.g., stated in Refs. [1,4] for the dynamic part of the precessing magnetization of Py. This would suggest that the pumped ac spin polarization should always be out of phase with the exciting dynamic magnetic component of the magnetization of the precessing magnetization. In that way both contributions would compensate themselves compensate to zero at all times and thus maintain the identical net-value as without pumping, i.e. with no precession of the magnetization at all. Observation (iii) is the most surprising. Obviously, the dynamic spin polarization is also laterally pumped into the $\mathrm{Co}: \mathrm{ZnO}$ since it appears to not only exist underneath the Py strip but also outside the region, which is covered by the strip, at least in the measured region (which is only 1 micron larger than the region of the strip). To underline that this small magnetic signal outside the strip region is not an experimental artifact, the circular polarization was flipped from $\sigma^{-}$(filled symbols) to $\sigma^{+}$(open symbols) as a control experiment; for Py, this has already been verified [23]. The results in Fig. 4 demonstrate that even outside the strip region the integrated contrast reverses at the $\mathrm{Co}_{3}$ edge, as expected 
for the XMCD effect. These findings corroborate that the rather noisy dynamic contrast at the Co edge in Fig. 3 is indeed a true dynamic magnetic contrast.

A different way of visualizing the dynamic magnetic contrast was introduced in Ref. [29]. For this kind of visualization the time dependence of the contrast of each pixel is fitted by a sine function. The obtained amplitude and phase of that point-by-point fit is plotted as an image; this is also shown in Fig. 4 for the $\mathrm{Ni}$ (c) and $\mathrm{Co}$ (d) $L_{3}$ edges. At the Ni $L_{3}$ edge the amplitude is maximal (white) within the strip with decreasing amplitude towards the edges, while it is close to zero (black) outside. The phase has a uniform value across the strip (homogeneously red), corroborating a quasiuniform excitation characteristic of the main FMR mode in a microstrip, i.e., all spins are precessing in phase $[25,28]$. Note that outside the region of the strip the derived phase values are not significant because of the very small amplitude of the signal. The situation is different for the Co $L_{3}$ edge, where the overall size of the signal is rather low and, thus, this visualization also appears rather noisy. Note that, while the amplitude scale for $\mathrm{Co}$ is a factor of ten smaller than for $\mathrm{Ni}$, the color scale of the phase is identical. Nonetheless, we see that, while the phase at the Ni edge is red, at Co it appears bluish, i.e., the observation is consistent with the opposite phase visible in the integral dynamic magnetic contrast. It should be noted that all visualizations (except the $\sigma^{+}$data) in Figs. 3 and 4 correspond to identical measurements at the $\mathrm{Ni}$ and Co edges, respectively. Unfortunately, in none of the different data visualizations presented so far can definite conclusions be drawn for the actual spatial distribution of the pumped spin polarization inside the $\mathrm{Co}: \mathrm{ZnO}$ layer. In particular, it is not possible to discriminate between noise and a lateral variation of the spin pumping efficiency, which could lead to interference effects within the pumped spin polarization with alternating contrast. The data can only provide a first evidence of a certain lateral extent of the pumped spin polarization outside the region of the strip.

An alternative approach of using the spatial resolution of STXM FMR for studying the lateral extent of the pumped spin polarization can be taken by exciting the Py strip nonuniformly. It has been predicted by micromagnetic simulations and verified by integral FMR measurements [25] that magnetic microstrips cannot only be excited quasiuniformly but also standing spin waves can exist due to the confined geometry. Their existence for the 50\% $\mathrm{Co}: \mathrm{ZnO} / \mathrm{Py}$ heterostructure has been verified here; see Fig. 2(a). These inhomogeneous excitations have already been observed in STXM-FMR experiments [27]; however, it turned out that these types of excitation can also have a nonstanding character [28]. In Fig. 5 the Py strip is excited with the identical microwave frequency of 9.477 $\mathrm{GHz}$, but the external field is increased to $106 \mathrm{mT}$ so that a quasistanding nonuniform excitation is observed. The three different regions of interest in Fig. 5 are selected such that they correspond to the maximum amplitude of the point-by-point fitted image given in (b). The corresponding phase image reveals only a moderate phase gradient across the strip, especially towards the ends, confirming the quasistanding character of this particular mode. We first discuss the data recorded at the $\mathrm{Ni} L_{3}$ edge. In Fig. 5(a) the integrated intensities of the dynamic magnetic contrast of the three regions of interest are shown for two consecutive precession cycles. A clear oscillatory behavior is visible with little phase difference for the three regions. Only the left part (green), which exhibits the strongest amplitude, is slightly phase shifted with respect to the two less intense maxima in the amplitude image. This can be interpreted as a slight inhomogeneity of the "proper" resonance condition along the strip. While the left part is nicely driven at resonance, the two other regions appear to be slightly off resonance and are thus slightly phase shifted and reduced in amplitude. This can be caused by a slight variation of the strip thickness, the edge roughness, or influences of local stray fields of unknown origin. The fact that local variations of the effective field can alter the behavior of the spin-wave modes has already been demonstrated with STXM FMR [28].

For the purpose of this work, the nonuniform excitation of the Py strip offers the possibility of investigating the spatial distribution of the pumped ac spin polarization inside the $\mathrm{Co}: \mathrm{ZnO}$ that is generated by an inhomogeneous distribution of the pumping across the Py strip. In Fig. 5(c) we show the integrated dynamic spin polarization for the three identical regions of interest recorded at the Co $L_{3}$ edge. Interestingly, in both the left (green) and right (blue) regions, where the amplitude of the dynamic magnetization component of the Py is maximum, the signal for Co is rather noisy and even a proper sinelike behavior is somewhat questionable. A sine fit of these two regions return somewhat reasonable values for the phase (roughly opposite to that of $\mathrm{Ni}$ ) and amplitude, which point towards a true dynamic magnetic contrast. However, in the left region (green) the phase $0 / 2 \pi$ is obviously a rather bad data point. In these regions we consider the fits of the dynamic magnetic contrast to be questionable which we indicate by the dotted lines. Therefore, the pumping efficiencies appear to be rather bad or low. It should be stressed that all three panels in Fig. 5(c) stem from the same timeresolved experimental image. Surprisingly, the quality of the fit is significantly improved for the center region (red). Here a rather clear oscillatory behavior of the pumped ac spin polarization can be seen for the Co edge, which fits a sine function rather satisfyingly. It is also out of phase with that measured at the $\mathrm{Ni}$ edge, like for the quasiuniform mode, and thus should represent a true dynamic magnetic contrast generated by spin pumping. It is remarkable that this rather clear signal can be seen at the Co $L_{3}$ edge in 
regions where the actual amplitude of the pumping itself as measured at the $\mathrm{Ni} L_{3}$ edge is rather weak. Also, this observation can be taken as indication of a lateral extent for the pumped ac spin polarization since it is likely that this region of the $\mathrm{Co}: \mathrm{ZnO}$ layer is not directly pumped by the Py above, where the amplitude of the precession is rather low. At least, the spatial distribution of the dynamic magnetic component of the ferromagnet exhibits no one-to-one correlation with the pumped ac spin polarization inside the $\mathrm{Co}: \mathrm{ZnO}$ layer.

Our findings for the spatial extent of the dynamic spin polarization measured inside the nonferromagnetic material with element specificity provide direct experimental evidence for spin pumping into an insulating oxide. Thus, they corroborate the interpretation that increased Gilbert damping seen in integral FMR in Fig. 1 is indeed a sign of spin pumping. The presence of spin pumping in a highly insulating paramagnetic oxide itself has already been indirectly reported [19] and led to two important observations. The pumping inferred from the FMR linewidth increases with increasing Co concentration. For a given concentration, the increase in FMR linewidth is maximum around the Néel temperature of the Co: $\mathrm{ZnO}$ layer. The fact that the uncompensated antiferromagnetic oxide exhibits signs of spin pumping even at room temperature suggests that the mechanism of transfer of angular momentum may be through local antiferromagnetic Co-O-Co correlations, which still exist at room temperature but are strongest close to the order temperature. We should note that at $50 \% \mathrm{Co:ZnO}$ most of these dopant configurations extend throughout the entire specimen and thus may also appear magnetically blocked on time scales of a FMR experiment, as pointed out in Ref. [19]. In addition to the evidence for spin pumping by utilizing the spatial resolution of the STXM FMR, we find that no one-to-one correspondence seems to exist with the spatial distribution of the dynamic behavior of the pumping ferromagnetic material. The results can be merely understood in the frame of a local pumping efficiency that generates the inhomogeneous appearance of pumped ac spin polarization. Considering that there is a lithographic step in between the deposition of the $\mathrm{Co}: \mathrm{ZnO}$ layer and Py, including a break of the vacuum, it is actually not surprising that the interface is not so well defined on an atomic scale as for the thin film heterostructure measured with conventional FMR in Fig. 1; for a transmission electron microscopy image of that interface, we refer the reader to Ref. [19]. Therefore, variations of the spin pumping efficiency, e.g., by scattering at defects introduced within the additional lithographic step, are actually not very surprising. Also,
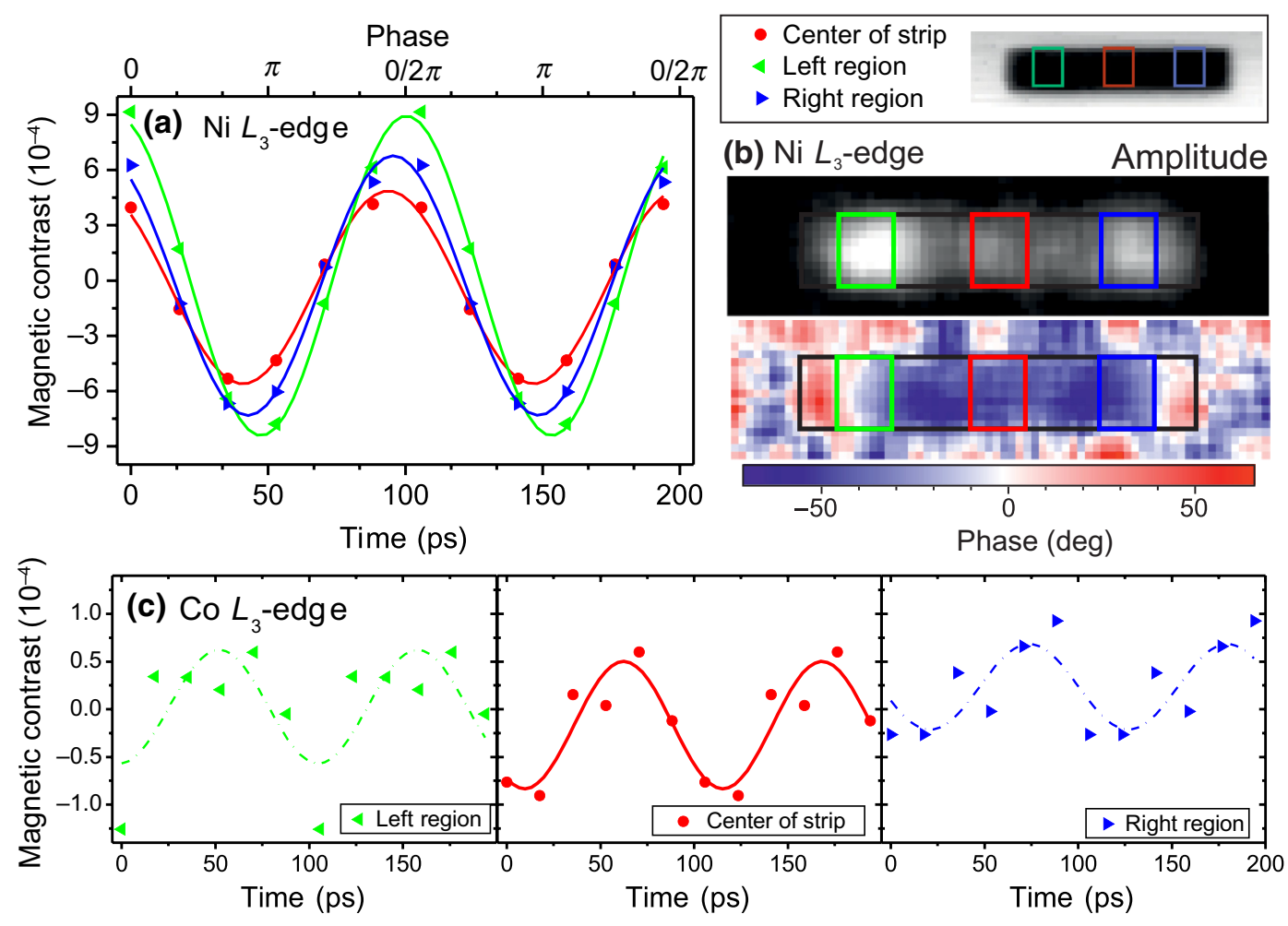

FIG. 5. (a) Integrated dynamic contrast for a nonuniform FMR excitation of the Py strip at $f_{\mathrm{MW}}=9.447 \mathrm{GHz}$ and $B_{\text {ext }}=106 \mathrm{mT}$ recorded at the Ni $L_{3}$ edge for the three regions of interest marked in the inset. In (b) the corresponding local amplitude and phase are shown. (c) Integrated dynamic magnetic contrast for the three regions of interest recorded at the Co $L_{3}$ edge. The position of the $1 \times 5 \mu \mathrm{m}^{2}$ large Py strip is indicated by the black frame. 
the large but finite Co-O-Co-dopant configurations mentioned before may play a role in understanding the laterally inhomogeneous pumping efficiency. Nevertheless, we find evidence that the pumped ac spin polarization can laterally spread out inside the nonferromagnetic material for finite distances, as indicated in Fig. 4. It even seems to form interference effects as suggested by the unexpectedly large and clear signal in the center of the strip despite the weak pumping in this very region, as seen in Fig. 5. The lateral extent of the pumped ac spin polarization is different from previous findings of the dc [15] and the ac [16] components in $\mathrm{Py} / \mathrm{Cu}$ heterostructures, where the polarization is confined to the area of the ferromagnet. A substantial lateral extent of the ac component of the pumped spin polarization inside the nonferromagnetic insulator can be supported by another consideration. Unlike in ISHE experiments, a high- $Z$ metallic material, like Pt or Pd, can be avoided and we can use $\mathrm{Co}: \mathrm{ZnO}$ as a highly insulating and rather low-Z material. Therefore, the spin relaxation times and/or the spin coherence length can be expected to be much larger than, for example, in metallic $\mathrm{Cu}$, where the expected decay length is of the order of $300 \mathrm{~nm}$ at $300 \mathrm{~K}$ [30] and is therefore not easily detected by the spatial resolution of the STXM $[15,16]$. In fact, long spin coherence lengths and times at room temperature are well known for $\mathrm{ZnO}$ [31] from optical measurements.

In summary, we demonstrate the feasibility of investigating the lateral distribution of the pumped ac spin polarization inside a $\mathrm{Co}: \mathrm{ZnO} / \mathrm{Py}$ heterostructure with element selectivity. A Py microstrip is driven into ferromagnetic resonance. Besides the main, quasiuniform exitation integral FMR als indicates the existence of quasistanding nonuniform excitations which both can be directly verified with STXM FMR. The dynamic spin polarization inside the adjacent nonferromagnet that is generated via spin pumping can be directly imaged with unprecedented spatio-temporal resolution. While the very existence of spin pumping as suggested by integral FMR studies can be unambiguously corroborated, the spatial extent of the spin polarization appears to be more complex and the findings are consistent with a locally varying spin pumping efficiency. More significantly, the dynamic spin polarization in the $\mathrm{Co}: \mathrm{ZnO}$ layer can be detected outside the region of the Py strip, thus inferring a laterally expanding spin polarization inside the nonferromagnet.

\section{ACKNOWLEDGMENTS}

The authors would like to thank the Austrian Science Foundation (FWF), Project No. I-3050, for financial support. Use of the Stanford Synchrotron Radiation Lightsource, SLAC National Accelerator Laboratory, is supported by the U.S. Department of Energy, Office of Science, Office of Basic Energy Sciences, under Contract No. DE-AC02-76SF00515. We gratefully acknowledge T.
Feggeler (University of Duisburg-Essen) for help during the SSRL beam time.

[1] Y. Tserkovnyak, A. Brataas, and G. E. W. Bauer, Enhanced Gilbert Damping in Thin Ferromagnetic Films, Phys. Rev. Lett. 88, 117601 (2002).

[2] H. Jiao and G. E. W. Bauer, Spin Backflow and ac Voltage Generation by Spin Pumping and the Inverse Spin Hall Effect, Phys. Rev. Lett. 110, 217602 (2013).

[3] D. Wei, M. Obstbaum, M. Ribow, C. H. Back, and G. Woltersdorf, Spin Hall voltages from a.c. and d.c. spin currents, Nat. Commun. 5, 3768 (2014).

[4] M. M. Qaid, T. Richter, A. Müller, C. Hauser, C. Ballani, and G. Schmidt, Radiation damping in ferromagnetic resonance induced by a conducting spin sink, Phys. Rev. B 96, 184405 (2017).

[5] K. Ando, S. Takahashi, J. Ieda, Y. Kajiwara, H. Nakayama, T. Yoshino, K. Harii, Y. Fujikawa, M. Matsuo, S. Maekawa, and E. Saitoh, Inverse spin-Hall effect induced by spin pumping in metallic system, J. Appl. Phys. 109, 103913 (2011).

[6] S. Geprägs, S. Meyer, S. Altmannshofer, M. Opel, F. Wilhelm, A. Rogalev, R. Gross, and S. T. B. Goennenwein, Investigation of induced $\mathrm{Pt}$ magnetic polarization in $\mathrm{Pt} / \mathrm{Y}_{3} \mathrm{Fe}_{5} \mathrm{O}_{1} 2$ bilayers, Appl. Phys. Lett. 101, 262407 (2012).

[7] J.-C. Rojas-Sanchez, M. Cubukcu, A. Jain, C. Vergnaud, C. Portemont, C. Ducruet, A. Barski, A. Marty, L. Vila, J.-P. Attane, E. Augendre, G. Desfonds, S. Gambarelli, H. Jaffres, J.-M. George, and M. Jamet, Spin pumping and inverse spin Hall effect in germanium, Phys. Rev. B 88, 064403 (2013).

[8] T. Richter, M. Paleschke, M. Wahler, F. Heyroth, H. Deniz, D. Hesse, and G. Schmidt, Spin pumping and inverse spin Hall effect in ultrathin $\mathrm{SrRuO}_{3}$ films around the percolation limit, Phys. Rev. B 96, 184407 (2017).

[9] J.-C. Lee, L.-W. Huang, D.-S. Hung, T.-H. Chiang, J. C. A. Huang, J.-Z. Liang, and S.-F. Lee, Inverse spin Hall effect induced by spin pumping into semiconducting $\mathrm{ZnO}$, Appl. Phys. Lett. 104, 052401 (2014).

[10] R. Urban, G. Woltersdorf, and B. Heinrich, Gilbert Damping in Single and Multilayer Ultrathin Films: Role of Interfaces in Nonlocal Spin Dynamics, Phys. Rev. Lett. 87, 217204 (2001).

[11] B. Heinrich, C. Burrowes, E. Montoya, B. Kardasz, E. Girt, Y.-Y. Song, Y. Sun, and M. Wu, Spin Pumping at the Magnetic Insulator (YIG)/Normal Metal (Au) Interfaces, Phys. Rev. Lett. 107, 066604 (2011).

[12] C. Hahn, G. de Loubens, M. Viret, O. Klein, V. V. Naletov, and J. Ben Youssef, Detection of Microwave Spin Pumping Using the Inverse Spin Hall Effect, Phys. Rev. Lett. 111, 217204 (2013)

[13] M. Weiler, J. M. Shaw, H. T. Nembach, and T. J. Silva, Phase-Sensitive Detection of Spin Pumping via the ac Inverse Spin Hall Effect, Phys. Rev. Lett. 113, 157204 (2014).

[14] S. Bonetti, R. Kukreja, Z. Chen, D. Spoddig, K. Ollefs, C. Schöppner, R. Meckenstock, A. Ney, J. Pinto, R. Houanche, 
J. Frisch, J. Stöhr, H. Dürr, and H. Ohldag, Microwave soft x-ray microscopy for nanoscale magnetization dynamics in the 5-10 GHz frequency range, Rev. Sci. Instrum. 86, 093703 (2015).

[15] R. Kukreja, S. Bonetti, Z. Chen, D. Backes, Y. Acremann, J. A. Katine, A. D. Kent, H. A. Dürr, H. Ohldag, and J. Stöhr, X-Ray Detection of Transient Magnetic Moments Induced by a Spin Current in $\mathrm{Cu}$, Phys. Rev. Lett. 115, 096601 (2015).

[16] J. Ding, W. Zhang, M. B. Jungfleisch, J. E. Pearson, H. Ohldag, V- Novosad, and A. Hoffmann, Direct observation of spin accumulation in $\mathrm{Cu}$ induced by spin pumping, Phys. Rev. Res. 2, 013262 (2020).

[17] J. Li, L. R. Shelford, P. Shafer, A. Tan, J. X. Deng, P. S. Keatley, C. Hwang, E. Arenholz, G. van der Laan, R. J. Hicken, and Z. Q. Qiu, Direct detection of pure ac spin current by X-ray pump-probe measurements, Phys. Rev. Lett 117, 076602 (2016).

[18] H. A. Dürr, T. Eimüller, H. J. Elmers, S. Eisebitt, M. Farle, W. Kuch, F. Matthes, M. Martins, H. C. Mertins, P. M. Oppeneer, L. Plucinski, C. M. Schneider, H. Wende, W. Wurth, and H. Zabel, A closer look into magnetism: Opportunities with synchrotron radiation, IEEE Transact. Magn. 45, 15 (2009).

[19] M. Buchner, J. Lumetzberger, V. Ney, T. Schaffers, N. Daffe, and A. Ney, Spin pumping from permalloy into uncompensated antiferromagnetic Co doped zinc oxide, J. Appl. Phys. 127, 043901 (2020).

[20] M. Buchner, B. Henne, V. Ney, and A. Ney, Transition from a hysteresis-like to an exchange-bias-like response of an uncompensated antiferromagnet, Phys. Rev. B 99, 064409 (2019).

[21] R. Narkowicz, D. Suter, and R. Stonies, Planar microresonators for EPR experiments, J. Magn. Reson. 175, 275 (2005).

[22] R. Narkowicz, D. Suter, and I. Niemeyer, Scaling of sensitivity and efficiency in planar microresonators for electron spin resonance, Rev. Sci. Instrum. 79, 084702 (2008).

[23] T. Schaffers, R. Meckenstock, D. Spoddig, T. Feggeler, K. Ollefs, C. Schöppner, S. Bonetti, H. Ohldag, M. Farle, and A. Ney, The combination of micro-resonators with spatially resolved ferromagnetic resonance, Rev. Sci. Instrum. 88, 093703 (2017).

[24] F. M. Römer, M. Möller, K. Wagner, L. Gathmann, R. Narkowicz, H. Zähres, B. R. Salles, P. Torelli, R. Meckenstock, J. Lindner, and M. Farle, In situ multifrequency ferromagnetic resonance and $\mathrm{x}$-ray magnetic circular dichroism investigations on $\mathrm{Fe} / \mathrm{GaAs}(110)$ : Enhanced g-factor, Appl. Phys. Lett. 100, 092402 (2012).

[25] A. Banholzer, R. Narkowicz, C. Hassel, R. Meckenstock, S. Stienen, O. Posth, D. Suter, M. Farle, and J. Lindner, Visualization of spin dynamics in single nanosized magnetic elements, Nanotechnol. 22, 295713 (2011).

[26] V. Ney, B. Henne, J. Lumetzberger, F. Wilhelm, K. Ollefs, A. Rogalev, A. Kovacs, M. Kieschnick, and A. Ney, Coalescence-driven magnetic order of the uncompensated antiferromagnetic Co doped ZnO, Phys. Rev. B 94, 224405 (2016).

[27] T. Schaffers, T. Feggeler, S. Pile, R. Meckenstock, M. Buchner, D. Spoddig, V. Ney, M. Farle, H. Wende, S. Wintz, M. Weigand, H. Ohldag, K. Ollefs, and A. Ney, Extracting the dynamic magnetic contrast in time-resolved X-ray transmission microscopy, Nanomaterials 9, 940 (2019).

[28] S. Pile, T. Feggeler, T. Schaffers, R. Meckenstock, M. Buchner, D. Spoddig, B. Zingsem, V. Ney, M. Farle, H. Wende, H. Ohldag, A. Ney, and K. Ollefs, Non-standing spin-waves in confined micrometer-sized ferromagnetic structures under uniform excitation, Appl. Phys. Lett. 116, 072401 (2020).

[29] F. Groß, N. Träger, J. Förster, M. Weigand, G. Schütz, and J. Gräfe, Nanoscale detection of spin wave deflection angles in permalloy, Appl. Phys. Lett. 114, 012406 (2019).

[30] P. Laczkowski, M. Cosset-Cheneau, W. Savero-Torres, V. T. Pham, G. Zahnd, H. Jaffres, N. Reyren, J.-C. RojasSanchez, A. Marty, L. Vila, J.-M. George, and J.-P. Attane, Spin-dependent transport characterization in metallic lateral spin valves using one-dimensional and threedimensional modeling, Phys. Rev. B 99, 134436 (2019).

[31] S. Ghosh, V. Sih, W. H. Lau, D. D. Awschalom, S.-Y. Bae, S. Wang, S. Vaidya, and G. Chapline, Room-temperature spin coherence in ZnO, Appl. Phys. Lett. 86, 232507 (2005). 